\title{
MOVEMENT OF THE FLAGELLA OF POLYTOMA UVELLA
}

\author{
BY C. J. BROKAW*
}

\author{
Division of Biology, California Institute of Technology, Pasadena, Califormia
}

\section{(Received 27 September 1962)}

\section{INTRODUCTION}

Hoffman-Berling (1955) and other workers have shown that flagella and cilia from a variety of organisms can be sensitized to adenosine triphosphate (ATP) by treatment with glycerol in a manner analogous to the preparation of glycerol-extracted muscle fibres. In most cases the movement obtained upon reactivation by ATP is a localized oscillating bending, without progression through the suspending medium (Bishop, 1962). On the other hand, studies of the movement of normal flagella have shown that active bending occurs along the length of the flagellum (Gray, 1955; Machin, 1958) and is somehow co-ordinated to yield propagated bending waves which produce a propulsive effect.

Flagella isolated from the alga, Polytoma uvella, by glycerol treatment have been reported previously to show propagated bending waves and forward progression when reactivated in media containing ATP (Brokaw, 1961, 1962). In the case of Polytoma, at least part of the co-ordinated bending of active elements along the length of the flagellum appears to survive the glycerol-isolation procedure, so that all the significant features of normal flagellar movement can be studied with this isolated flagella preparation. A more detailed analysis of these movements, based on photographic records of the movement of isolated flagella, is given in the present paper. Photographs of flagella on intact cells of Polytoma have been included for comparison, since previous descriptions of the movements of flagella of Polytoma (Ulehla, I9II; Lowndes, 194I) were not based on identical methods of observation.

\section{METHODS}

Flagella were isolated from Polytoma uvella by differential centrifugation after mixing the cells with 3-4 volumes of a $70 \%$ glycerol solution containing $0.01 \mathrm{M}-\mathrm{MgCl}_{2}$ and $0.02 \mathrm{M}$ tris-thioglycolate buffer at $\mathrm{pH} 7 \cdot 8$, as described previously (Brokaw, $196 \mathrm{r}$ ). Reactivation was observed at $12^{\circ} \mathrm{C}$ in a medium containing $0.2 \mathrm{M}$ tris-thioglycolate buffer, $\mathrm{pH} 7.8,0.05 \mathrm{M}-\mathrm{KCl}, 0.004 \mathrm{M}-\mathrm{MgCl}_{2}, 2.5 \%$ polyvinylpyrollidinone and $\mathrm{I}-2 \times \mathrm{IO}^{-4} \mathrm{M}-\mathrm{ATP}$.

Photomicrographs and stroboscopic observations were made using dark-field illumination with a General Electric FT-230 short-gap flash lamp, operated at I500-

- Much of the work reported here was carried out in the Department of Zoology at the University of Minnesota, with support from the United States Public Health Service (RG-7208) and the Graduate School of the University of Minnesota. At the California Institute of Technology the work has been supported by the United States Public Health Service (RG-6965) and the Max C.Fleischmann Foundation of Nevada. 
2300 V. d.c. For operation as a stroboscope, a flash capacitor of 0.1 or $0.25 \mathrm{mfd}$. was used, and continuous operation was possible at frequencies up to $60 \mathrm{per}$ sec. For photomicrography, a flash capacitor of 5 or $10 \mathrm{mfd}$. was used, and a $200 \mathrm{mfd}$. storage capacitor was used in parallel with the power supply to recharge the flash capacitor rapidly for multiple flash exposures.

The flash lamp was triggered by a General Radio I531-A 'Strobotac' calibrated against the 60 cycle a.c. power supply, with the output trigger capacitance increased to $0.07 \mathrm{mfd}$. A burst of trigger pulses for multiple flash exposures was obtained by controlling the 'Strobotac' by grounding the control grid of its thyratron tube (V 2) through a $0.02 \mathrm{mfd}$. capacitor and the normally closed contacts of a sensitive plate relay. The relay was opened for a predetermined interval by the gate output of a Tektronix type 162 wave-form generator, triggered by the shutter contacts of the camera. Photographs were taken with a Robot Star II automatic $35 \mathrm{~mm}$. camera, on Kodak Tri-X film, with a magnification on film of $320 \times$.

\section{OBSERVATIONS}

\section{Intact cells}

Two technical difficulties limit the quality of the photographs which can be obtained of the patterns of beating of flagella on intact cells. With dark-field illumination at sufficient intensity to photograph the flagella, the cell body reflects so much light that the basal portions of the flagella are obscured by the over-exposed image of the cell body. Secondly, swimming cells normally rotate continually about a longitudinal axis, so that the chance of the flagella remaining in the focal plane of the microscope long enough for observation, focusing and photography is negligible under normal conditions. By using a very thin layer of suspending medium between the slide and the cover glass, the rotation and forward progress of the cells were restricted enough to permit photography, but the cells did not remain motile for more than a few minutes under these conditions, and consequently their flagellar movement may not be entirely normal. Photographs were taken after concentrating cells in a small quantity of culture medium by mild centrifugation. The microscope stage was maintained at a temperature of about $14^{\circ} \mathrm{C}$.

Only a few photographs were obtained in which both flagella on a cell were in focus; one of these is shown in Pl. I, fig. I. More complete wave patterns are shown in Pl. I, figs. 2-4. The wave patterns in these photographs appear identical to those observed by means of stroboscopic illumination on cells swimming in deeper layers of suspending medium. Direct observation of cells which are free to rotate also indicates that the waves of flagellar bending are predominantly planar.

Bending waves originate near the base of the flagellum and pass distally with little, if any, decrement in velocity or amplitude. The amplitude of bending sometimes appears to be greater when the flagellum is bending towards the cell than when it is bending away from the cell. The somewhat asymmetrical waves which result may be more efficient in forward propulsion of the cell, but the major propulsive effect will be in the direction of wave travel along the flagellum.

The beat frequencies of flagella, on cells under these conditions, are around ro-20 per sec. Higher beat frequencies can be obtained by removing calcium ions from the 
medium and by working at higher temperatures. At $25^{\circ} \mathrm{C}$. in the presence of $0.5 \mathrm{~mm}$ ethylenediamine-tetraacetate (buffered at $\mathrm{pH} 7{ }^{\circ}$ ) beat frequencies average around $5^{\circ}$ per sec. and may occasionally exceed 60 per sec. There is usually little correlation between the frequency of beat of the two flagella on a cell. The frequency of one flagellum may be $50 \%$ greater than that of the other when both appear to be beating normally, and the extreme case where one flagellum is beating at a normal rate and the other flagellum is motionless is frequently seen. Such differences in frequency are probably partially responsible for the asymmetry which causes the normal swimming path of the cells to be helical.

Normally the flagella turn sharply just after they emerge from the anterior end of the cell, so that their propulsive effort is directed backwards to produce forward locomotion. In the presence of calcium ions, frequent 'shock reactions' occur, in which the flagella are thrown forward and beat in this position for $2-3 \mathrm{sec}$., causing the cell to dart backwards (Pl. I, fig. 5).

Beat patterns of flagella on cells swimming in media to which methyl cellulose has been added to increase the viscosity are shown in Pl. I, figs. 6-8. These patterns show decreased amplitude and wavelength, and these effects become greater at slightly higher concentrations of methyl cellulose. However, the primary effect of methyl cellulose is a decrease in beat frequency. These photographs were taken at flash rates of 3-5 per sec., and the beat frequencies are around $\mathbf{I}-\mathbf{2}$ per sec. The wave pattern shown in Pl. I, fig. 8, with significantly reduced amplitude and wavelength, most nearly resembles the almost sinusoidal wave patterns of sea urchin sperm tails (Gray, I955) and clearly implies the presence of active bending along the length of the flagellum to maintain a constant amplitude of bending. In general, the wave patterns observed in the presence of methyl cellulose at beat frequencies around 2 per sec. are not obviously different from those observed with stroboscopic illumination when the flagella are beating at $50-60$ per sec.

\section{Isolated flagella}

Beating most nearly resembling that of normal flagella on cells is seen when isolated flagella become attached to the slide or cover glass by their basal ends, as shown in Pl. 2, figs. I-3. Under these conditions, the beat frequencies of the isolated flagella (about 10-12 per sec.) are somewhat less than those of normal flagella. The amplitude of bending of the isolated flagella is less than that of normal flagella on cells, but in other respects the beat patterns resemble those of normal flagella. There is no obvious damping of the amplitude of bending as the waves pass distally; in some cases the amplitude of bending increases along the flagellum. The bending waves always pass from the bent, basal end of the flagellum towards the thinner, distal end, even when attachment of both ends to the slide imposes symmetrical mechanical boundary conditions (Pl. 2, fig. 4) or when only the distal end is attached. These flagella frequently rotate slowly around their point of attachment, always in the direction indicated in Text-fig. $\mathrm{x} a$.

When a relatively thick layer of suspending medium separates the slide and cover glass, some flagella become attached to the slide nearly vertically. When the beating is viewed along the longitudinal axis of the flagellum, patterns such as those sketched in Text-fig. $\mathrm{I} b$ are seen. Since the flagella pass nearly through the centre of these 
patterns, they are beating essentially in a plane which rotates around the longitudinal axis every 3 or 4 beats. Circular patterns are never observed.

Flagella which are not attached to the slide move forward about $\mathrm{I} \mu$ each time a wave of bending passes along the flagellum, so that they swim through the medium, basal end first. Because of their forward movement and rotation, they are difficult to photograph clearly, but some information can be obtained from the records in Pl. 2, figs. $5^{-8}$. These photographs suggest that active bending can occur along at least half of the length of the flagellum, and at different times, but this interpretation is complicated by the rotation of the flagellum which also occurs during the beat.

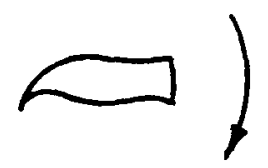

a

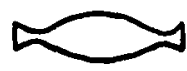

c

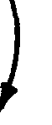

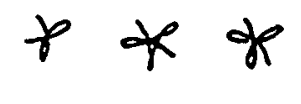

$b$

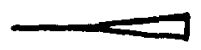

$d$

Text-fig. I. (a) Typical envelope of beat of an isolated flagellum attached to a slide with the plane of beat parallel to the slide. The arrow indicates the direction of rotation around the point of attachment. (b) Typical patterns seen when a beating fiagellum is viewed along its length. (c) Typical envelope of beat of a free-swimming flagellum. (d) Typical envelope of beat of a flagellum attached to a slide with the principal plane of beat perpendicular to the slide.

Visual observation of rapidly swimming flagella gives an impression similar to Text-fig. I $c$, with nodes near each end of the envelope of the beat. The paths of swimming flagella are not noticeably helical, suggesting that the beat is nearly symmetrical.

In the presence of enough methyl cellulose to reduce the beat frequencies to about I-2 per sec., the wave patterns of many attached flagella are only slightly altered, in agreement with the observations on intact cells (Pl. 2, figs. 9-I I). Patterns indicating considerable damping of wave amplitude are also observed, as in P1. 2, fig. 12.

One feature of the beating can be seen more clearly when the beat frequency is reduced with methyl cellulose. Flagella become attached to the slide in such a manner that the basal bend of the flagellum is not visible because it is oriented in a plane perpendicular to the slide. Longitudinal movement of the basal region of the flagellum can be seen, but lateral bending is apparent only near the tip of the flagellum, as sketched in Text-fig. I $d$. This suggests that the plane of bending twists somewhat as the bending waves pass along a flagellum, perhaps explaining the rotation of the flagellum when it is free.

If the beat frequency is lowered to $\mathrm{I}-2$ per sec. by decreasing the ATP concentration to $\mathrm{I}-2 \times 10^{-5} \mathrm{M}$, many flagella are found beating abnormally, as shown in Pl. 2, figs. 13-15. Such patterns are occasionally seen at higher ATP concentrations, but 
have never been observed when the beat frequency was reduced to I-2 per sec. by adding methyl cellulose.

At still lower ATP concentrations, the rhythm of the beating may become irregular, with pauses in one or both of the extreme lateral positions. Significantly, the flagellum does not always straighten out in these lateral positions, but remains bent into an $\mathrm{S}$-shape, as shown in Pl. 2, fig. I 5. Motionless flagella with S-shaped bends are often seen, but they are never seen bent into an ' $O$ ' of equal radius of curvature. The bending elements along the length of the flagellum therefore may not be independent.

\section{DISCUSSION}

The basic similarity between the patterns of bending waves in glycerol-isolated and normal flagella of Polytoma and in other flagella which have been critically examined (Gray, 1955, 1958) suggests that they are generated by similar mechanisms involving co-ordinated activity of bending elements distributed along the flagella. The differences which do appear between isolated and normal Polytoma flagella can be interpreted as a general reduction in the amount of active bending following glycerol treatment.

The alternative interpretation of the bending waves of isolated flagella would suggest that only a limited region of the flagellum bends actively, and that within this region all elements bend in the same phase. Wave propagation beyond this region would be strictly passive, as formulated by Machin (1958). Decreasing amplitude of the propagated waves would be expected, but would be difficult to detect in such short flagella. No proof that this model would explain the forward swimming of isolated flagella has been found; certainly swimming could not result if the region of active bending was centred between the ends of a flagellum with uniform mechanical properties. However, this localization is implied by the shape of the envelopes of swimming flagella (Text-fig. I $c$ ) and the frequent observation of maximum amplitude near the mid-region of attached flagella (e.g. Pl. 2, figs. 4-6). The patterns of the bending waves which have been photographed appear to exclude this alternative interpretation and indicate that active bending elements are operating in different phases along much of the length of a flagellum, after isolation as well as under normal conditions.

The difference between the movements of glycerol-isolated Polytoma flagella and the movements of glycerol-treated spermatozoa reported by other workers is not simply the result of differences in methods. Whole sea-urchin (Strongylocentrotus purpuratus) spermatozoa treated with solutions identical to those used for Polytoma showed vigorous beating, but wave propagation and forward swimming were insignificant. More vigorous mechanical treatment was required to separate the sperm tails from their heads, and the isolated tails showed practically no movement in the presence of ATP (Brokaw, unpublished). We have also found some biochemical differences between flagella from Polytoma and sea-urchin spermatozoa.

The photographs of the wave patterns of flagella in the presence and absence of methyl cellulose confirm earlier observations that the beat frequency is much more sensitive to variations in viscosity than the amplitude or wavelength of beat. This behaviour is unlike that of most simple oscillatory systems. It may be partially explained by the following analysis. 
The velocity of forward propulsion, $V$, resulting from sinusoidal bending waves passing along a flagellum has been formulated by Gray \& Hancock (1955). For an isolated flagellum,

$$
V=\frac{V_{w} s^{2}}{2\left(\mathrm{I}+s^{2}\right)}
$$

where $V_{w}=$ wave velocity relative to a point on the flagellum, and $s=$ wave 'shape' $=2 \pi b / \lambda$, where $b=$ wave amplitude and $\lambda=$ wavelength. Several equations have been formulated for the power dissipated against the viscous resistance of the medium during flagellar movement. The one given by Rothschild ( $196 \mathrm{I}$ ) can be simplified to an approximate form which states that the power dissipation, $P$, will be proportional to

$$
\frac{V_{w}^{2} s^{2}}{\mathrm{I}+\pi s / 4}
$$

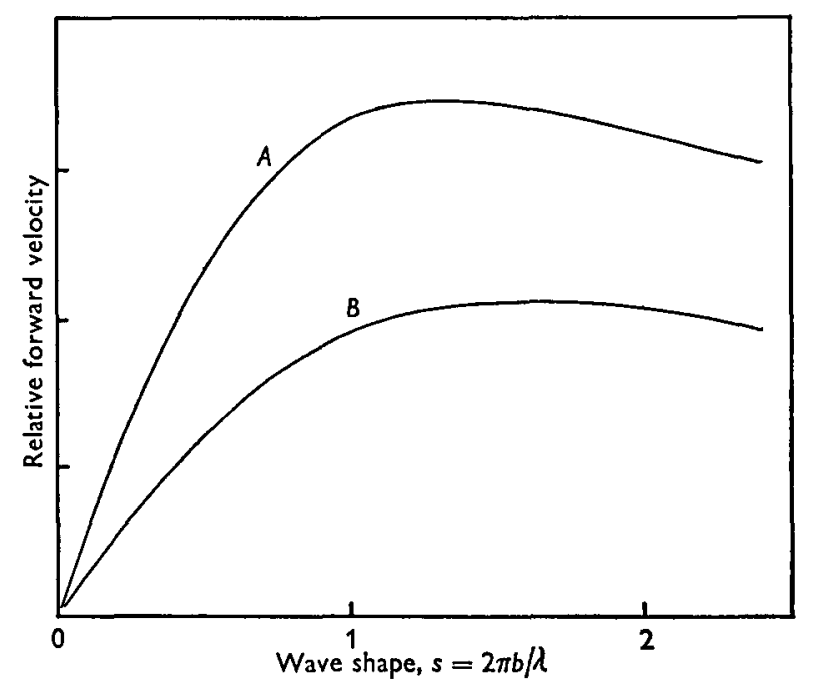

Text-fig. 2. Curves showing the theoretical effect of wave shape, $s$, on the forward velocity of flagellar movement when the power dissipation against viscous resistances is constant. $A=$ an isolated flagellum. $B=$ a flagellum propelling a relatively large cell.

By eliminating $V_{w}$ between these two expressions, we find that the forward velocity is proportional to

$$
\frac{P^{\frac{1}{2}} s(\mathrm{I}+\pi s / 4)^{\frac{1}{2}}}{\mathrm{I}+s^{2}} .
$$

The behaviour of this function when $P$ is constant is illustrated in Text-fig. 2, curve $A$. There will be an optimum wave shape which is independent of power or viscosity, corresponding to $s=$ about $\mathrm{I} \cdot 2$. As long as $s$ remains in the range of $\mathrm{I}-2$, there will be little variation in efficiency. A rough indication of the effect of the presence of a cell body comparable in size to that of Polytoma (radius $=5 \mu$ ) is illustrated by curve $B$, obtained by using equation XXVI of Gray \& Hancock (1955) for the forward velocity. The presence of the cell body has little effect on the general behaviour of the function, but will shift the optimum range to somewhat greater values of $s$.

For sea-urchin spermatozoa, with an almost negligible head, measurements made by 
Gray (1955) indicate a value of $s=\mathrm{I} \cdot 05$. Measurements on a few of the photographs of normal Polytoma indicate values of $s$ averaging around $\mathrm{I} \cdot \mathrm{g}$.

This analysis suggests that, for optimum efficiency in forward propulsion of cells, the mechanism of flagellar beating should maintain a wave amplitude corresponding to values of $s$ in the range of $\mathrm{I}-2$, and adjust to variations in power availability (or equivalent variations in viscosity) by varying the wave velocity, $V_{w}$. The wave velocity is equal to the product of beat frequency and wavelength. The wavelength will depend on the mechanism by which bending waves are propagated along the flagellum. If, as now seems most likely, propagation is primarily mechanical, the wavelength will be directly related to a characteristic length constant, $l_{0}$, determined by the physical conditions for bending. Since $l_{0}$ is proportional to the fourth root of

$$
\frac{\text { elastic modulus of flagellum }}{\text { frequency } \times \text { viscosity }}
$$

(Machin, 1958), the wavelength will be relatively insensitive to altered conditions. If a different type of propagation mechanism is involved, an extra expenditure of effort will be required to give a wavelength other than that determined by the physical conditions. This conclusion is also expressed in an equation for the optimum conditions for forward swimming developed by Machin (1958). If the wavelength is determined by these considerations, maximum propulsive efficiency can be achieved only by varying the frequency of beat to compensate for different conditions.

At the high methyl cellulose concentrations used to obtain the patterns in Pl. 2, figs. $6-8$, there is some reduction in $s$, but $s$ remains greater than $I$, and the reduction in beat frequency is always much greater.

With isolated flagella, values of $s$ less than I are commonly observed, both at normal and increased viscosities. If there is a mechanism for maintaining $s$ near an optimum value, it appears not to be an essential part of the mechanism of beating.

The wave patterns seen when the frequency of beat of isolated flagella is lowered by reducing the ATP concentration are unlike those observed in media of high viscosity. This difference is inconsistent with the suggestion that the effect of ATP on beat frequency is a 'plasticizing' effect equivalent to a change in the 'internal viscosity' of the flagellum, and agrees with an earlier conclusion based on less direct evidence (Brokaw, 1962). An effect of ATP on the amount of power available for beating would be equivalent to a change in viscosity and is also excluded. The wave patterns obtained at low ATP concentrations suggest that under these conditions the mechanism responsible for producing normal nearly sinusoidal bending waves functions imperfectly. Further study of wave patterns under these and other conditions may provide some clues to the properties of this co-ordination mechanism.

\section{SUMMARY}

I. Dark-field photographic records of the wave patterns of moving flagella have been made using multiple-flash exposures at flash rates of up to $5^{\circ}$ per sec. Patterns obtained from ATP-reactivated isolated flagella show reduced amplitude of bending, but are otherwise similar to those obtained from flagella under normal conditions. The co-ordination required to produce propagated waves of active bending appears to be preserved after isolation and reactivation. 
2. Addition of methyl cellulose to the medium to increase the viscosity reduces the frequency of beat much more than the amplitude. This behaviour can be partially explained by an analysis of the equations for sinusoidal wave movement of flagella which shows that maximum efficiency of forward swimming will be obtained if the amplitude of beat is maintained greater than $\frac{1}{2} \pi$ times the wavelength, and variations in available power or viscosity are compensated by changes in beat frequency.

3. Wave patterns at low frequencies in low ATP concentrations are unlike those obtained when the frequency is reduced by increased viscosity. The effect of ATP concentration on beat frequency is not explained by an effect on the power available for beating or by an effect on the 'internal viscosity' of the flagella.

\section{REFERENCES}

Bishop, D. W. (1962). Sperm motility. Physiol. Rev. 42, 1-59.

Brokaw, C. J. (r96r). Movement and nucleoside polyphosphatase activity of isolated flagella from Polytoma uvella. Exp. Cell Res. 22, 15 1-62.

Brokaw, C. J. (1962). Studies on isolated flagella. In: Spermatozoan Motility, Bishop, D. W., ed., pp. 269-78. American Association for the Advancement of Science.

GrAY, J. (1955). The movement of sea-urchin spermatozoa. F. Exp. Biol. 32, 775-801.

Gray, J. (1958). The movement of the spermatozoa of the bull. $\mathscr{F}$. Exp. Biol. 35, 96-108.

Gray, J. \& HaNcock, G. J. (1955). The propulsion of sea-urchin spermatozoa. F. Exp. Biol. 32, 802-14.

Hoffman-Berling, H. (1955). Geisselmodelle und Adenosintriphosphat (ATP). Biochim. Biophys. Acta, 16, 146-54.

Lowndes, A. G. (1941). On flagellar movement in unicellular organisms. Proc. Zool. Soc. Lond. IIIA, I I I-34.

Machin, K. E. (1958). Wave propagation along flagella. F. Exp. Biol. 35, 796-806.

ROTHSCHILD, LORD (1961). Sperm energetics. An account of work in progress. In: The Cell and the Organism, Ramsay, J. A. \& Wigglesworth, V. B., eds., pp. 9-21. Cambridge University Press.

UlehlA, V. (1911). Ultramikroskopische Studien über Geisselbewegung. Biol. Zbl. 31, 689-705.

\section{EXPLANATION OF PLATES}

Plate I

Multiple-flash photographs of flagella attached to cells of Polytoma uvella.

Fig. I. Flash rate 50 per sec.

Figs. 2-5. Flash rate 40 per sec.

Fig. 6. Flash rate 5 per sec., methyl cellulose added to the medium.

Figs 7 and 8. Flash rate 3 per sec., methyl cellulose added to the medium.

\section{Plate 2}

Multiple-flash photographs of isolated flagella from Polytoma uvella reactivated in the presence of ATP

Figs. 1-4. Flash rate 50 per sec.

Figs. 5-8. Flash rate 20 per sec., with a small amount of methyl cellulose added to the medium.

Fig. 9. Flash rate 5 per sec., methyl cellulose added to the medium.

Figs. 10-1 2. Flash rate 8 per sec., methyl cellulose added to the medium.

Figs. 13-15. Flash rate 5 per sec., low ATP concentration. 


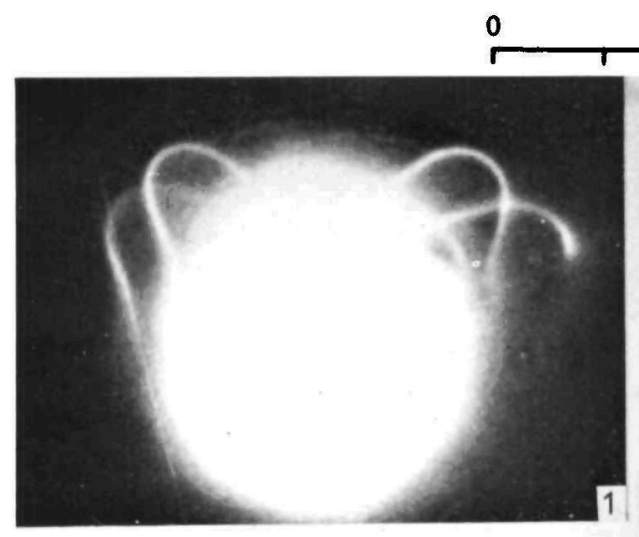

$\stackrel{15}{\mu}^{2}$
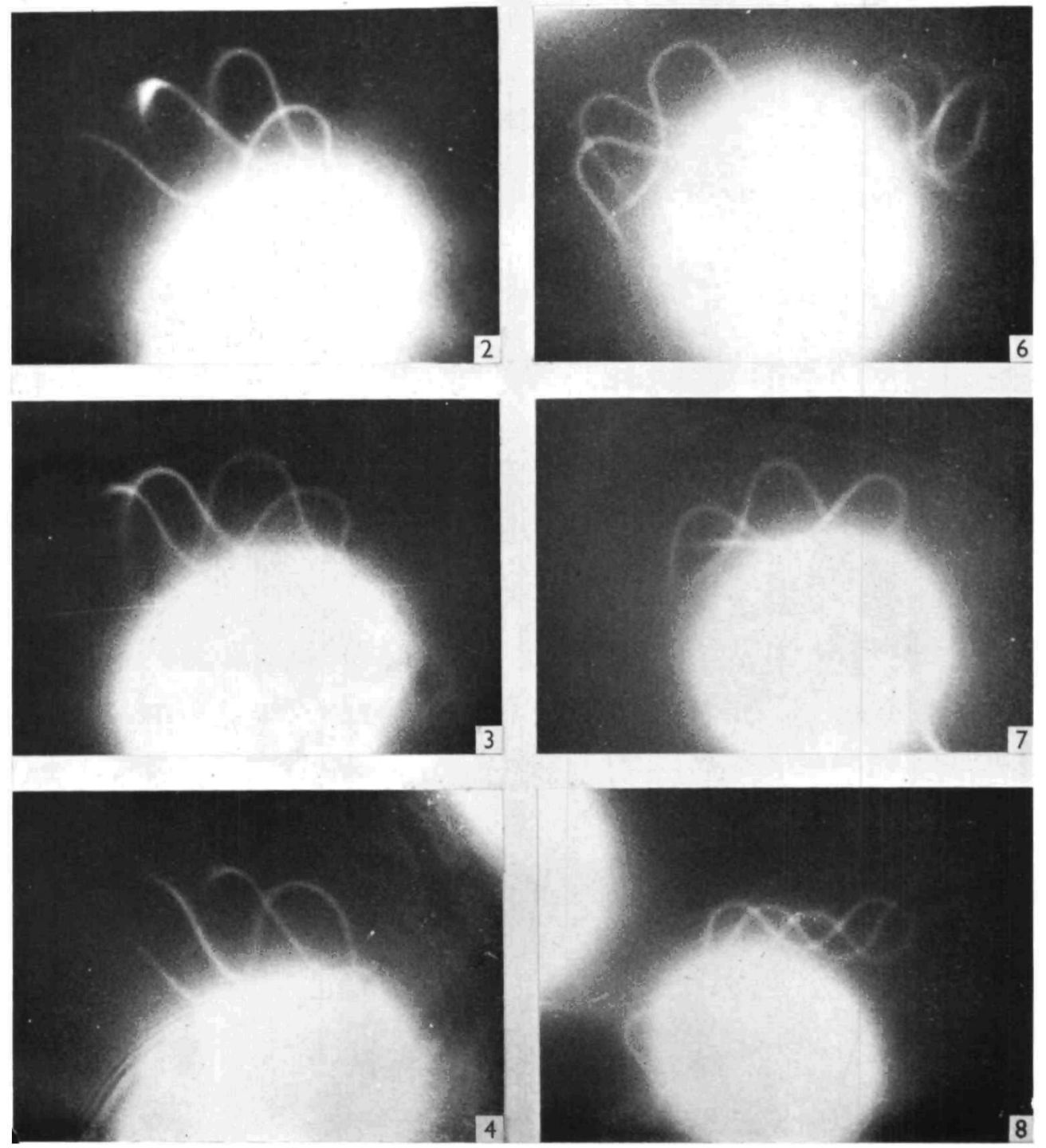

C. J. BROKAW

(Facing p. 156) 

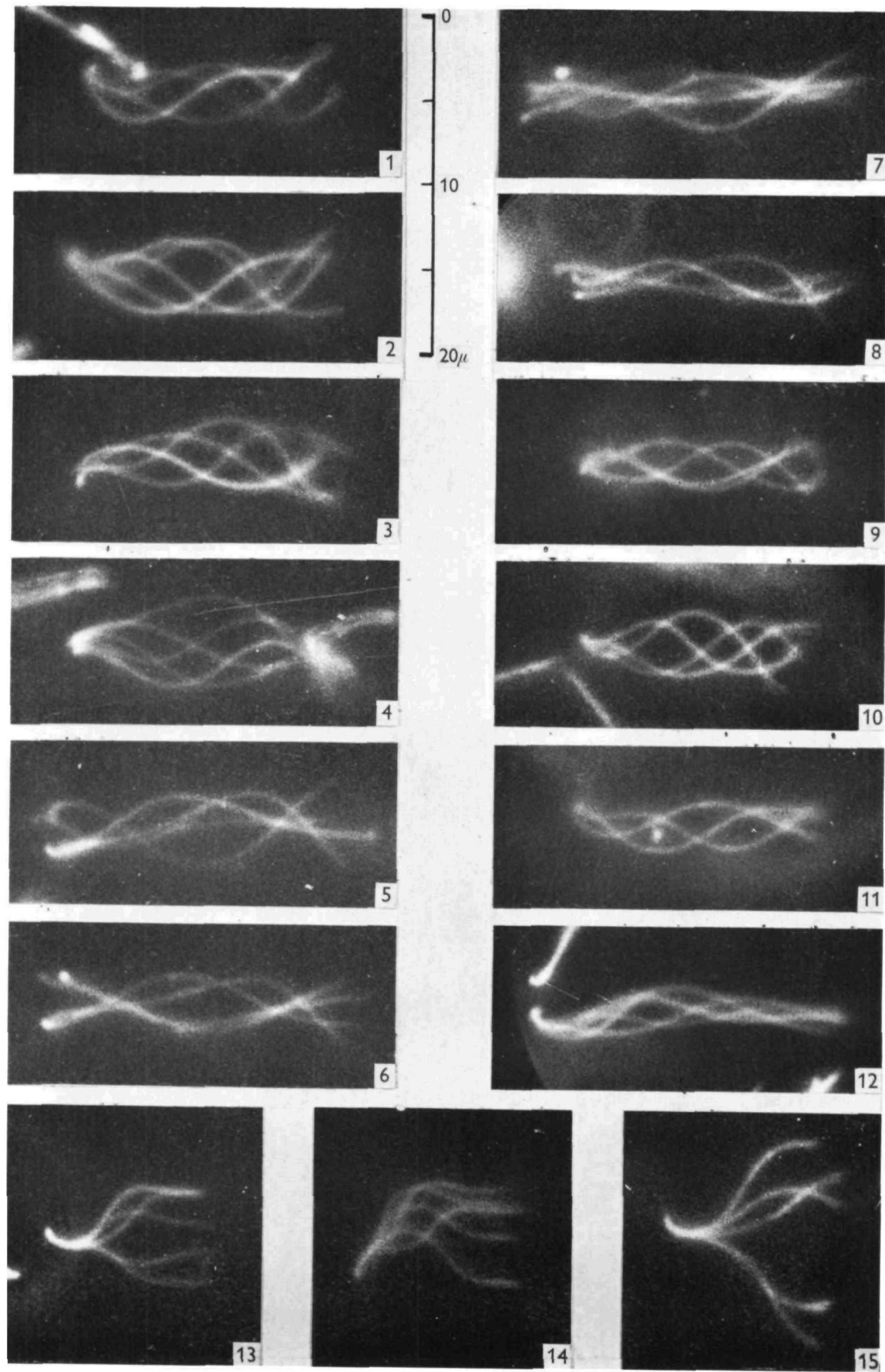

C. J BROKAW 